\title{
Anterolateral Minimally Invasive Total Hip Arthroplasty: Pitfalls During the Learning Curve and Clinical Outcomes
}

Christos KOUTSERIMPAS, Konstantinos RAPTIS, Despina TSAKALOU, Ilias KARAISKOS, Konstantinos KOURELIS

Department of Orthopaedics and Traumatology, "251" Hellenic Air Force General Hospital of Athens, Greece

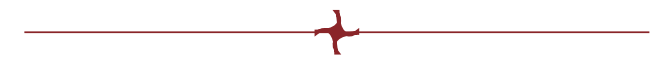

\begin{abstract}
Introduction: To report outcomes and complications from a modified anterolateral minimal invasive surgery (ALMIS) approach for total hip arthroplasty (THA).

Patients and methods: All patients undergoing THA through ALMIS approach during a seven-year period were retrospectively evaluated.

Results: One hundred fifty-four patients with a mean follow-up of 4.6 years were studied. The mean Harris hip score was found to be 91.1. A number of complications were recorded, including two cases (1.3\%) of greater avulsion trochanter, one case $(0.6 \%)$ of femur fractures, $12(7.8 \%)$ cases of Trendelenburg sign and 12 cases of heterotopic ossification.

Conclusions: This approach offers good visualization during acetabulum and femur preparation and excellent clinical results during mid-term follow-up.
\end{abstract}

Keywords: anterolateral hip, minimal invasive surgery, total hip arthroplasty, hip reconstruction, minimal hip, arthroplasty.

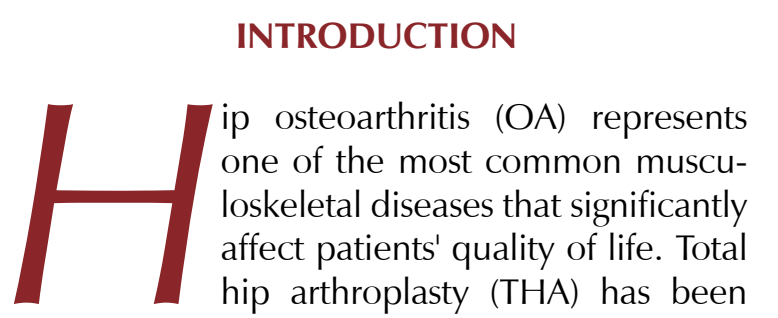
considered the gold standard in the treatment of end-stage $\mathrm{OA}$ of this joint and one of the most effective operations in modern surgery (1). Total hip arthroplasty may be performed through a variety of surgical approaches, each of them with its own well-documented benefits and risks $(1,2)$.

During the last two decades, numerous minimally invasive (MIS) hip approaches have been introduced in order to minimize soft tissue damage and therefore, to reduce hemorrhage, as well as lead to faster and possible better clinical

\footnotetext{
Address for correspondence:

C. Koutserimpas, $M D$

Department of Orthopaedics and Traumatology,"251" Hellenic Air Force General Hospital of Athens, Greece

Postal address: Kanellopoulou Av, Athens 11525, Greece

Tel: 00306948712130

Email:chrisku91@hotmail.com

ORCID: 0000-0002-1398-9626
} 
outcomes. It is believed that MIS approaches may reduce the length of hospital stay (LOS), recovery period and surgery-related pain (1-3).

In our department we utilize a modified anterolateral MIS (ALMIS) approach for THA, as described by Christodoulou in 2017 (3-5). The purpose of this study is to revisit intraoperative tips and tricks, report fallpits during the learning curve of the approach and present both midterm clinical outcomes and complications.

\section{MATERIAL AND METHODS}

This his is a retrospective observational study of a prospectively maintained database. All patients suffering from hip $\mathrm{OA}$ and treated surgically at the Orthopaedics and Traumatology Department of "251" Hellenic Air Force General Hospital of Athens, Greece, with the ALMIS approach, from January 2013 until December 2019, were eligible for this study. Revision cases were excluded.

Patients' demographics, including gender and age, as well as LOS and transfusion rate were evaluated from the patients' medical records. During follow-up, Harris hip score and complications that occurred intraoperatively (femoral shaft and greater trochanter fractures), early postoperatively [dislocation, superficial hematoma, superficial infection, leg lengthening $(>1.5 \mathrm{~cm})$ and Trendelenburg sign] and late postoperatively [dislocation, heterotopic ossification (Brooker scale $>$ III)] were recorded.

The present study has been approved by the hospital's bioethics committee (approval number: $\Phi .400 / 11 / 406168 / \Sigma .374)$.

\section{Surgical technique}

Under general, subarachnoid or epidural anesthesia, the patient is placed in lateral decubitus position, while the contra-lateral greater trochanter is positioned at the edge of the surgical table. The contra-lateral leg is stabilized on the posterior leg support, while the posterior support is extended 20 degrees and slightly abducted, enabling better mobilization and access during femoral preparation. The anterior leg support is removed. (Figure 1 ).

A longitudinal incision, about $8 \mathrm{~cm}(4 \mathrm{~cm}$ proximally and $4 \mathrm{~cm}$ distally of the greater trochanter tip), is performed right above the greater trochanter region (Figure 2A). The aponevrosis of

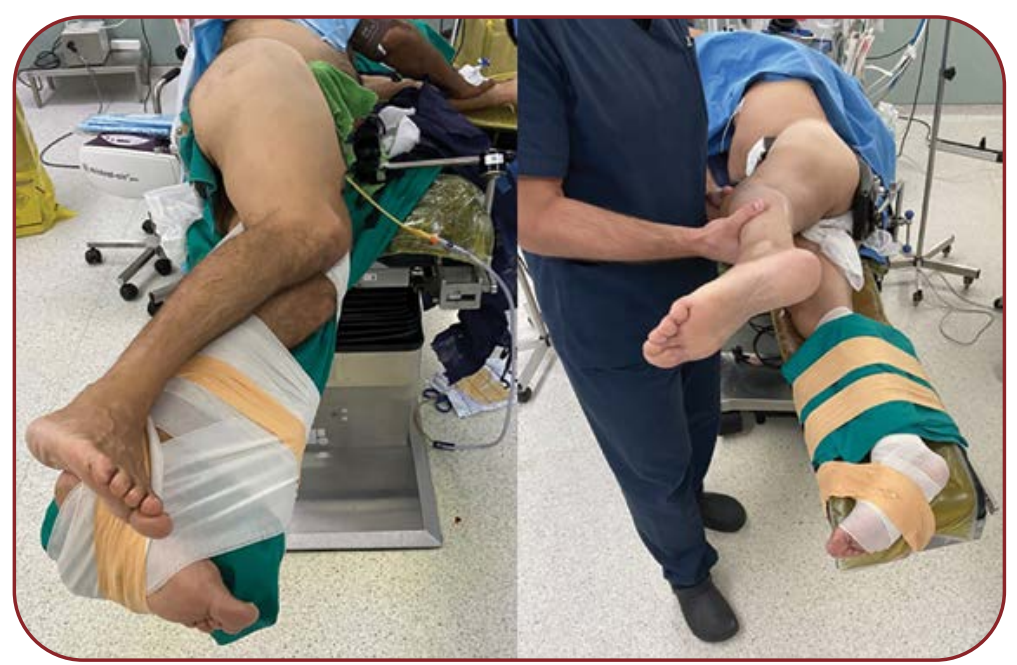

FIGURE 1. Positioning of the patient. The posterior support is extended about 20 degrees and slightly abducted, while the anterior leg support is removed, enabling better access during femoral preparation

the tensor fascia latae is curved and splitted proximally towards the anterior iliac spine and distally to the longitudinal femur axis (Figure 2B). At this point, the interval between tensor fascia latae, gluteus medius and vastus lateralis is exposed with the use of a blunt and then a sharp curved Hohmann retractor, which is placed towards the acetabulum roof (Figure 2C, D). With the use of electrocoagulation, few anterior gluteus medius fibers, as well as gluteus minimus, found directly under the gluteus medius' anterior fibers are elevated and held by sutures to be controlled during the operation (Figure 2E, F). In this surgical field, normally, no vessel branch is at risk; therefore, this approach is relatively simple and bloodless.

The limb is held in external rotation and a reversed " $\mathrm{T}$ " capsulotomy is performed. To avoid bleeding, the incision at the base of the greater trochanter should not be extended to the vastus lateralis fibers. Femoral dislocation using careful traction and external rotation by a screwdriver handle (tirebusson) inserted to the usually sclerotic anterolateral femoral neck-head junction is performed (Figure $2 \mathrm{G}, \mathrm{H}$ ). The dislocated neck is cut usually $1-1.5 \mathrm{~cm}$ proximally of the lesser trochanter and at an angle of approximately $45^{\circ}$ relative to the long axis of the femur and at an antevertion angle at about $10^{\circ}$ (according to the preoperative planning) (Figure 2l). In difficult cases such as those of acetabular protrusion that 


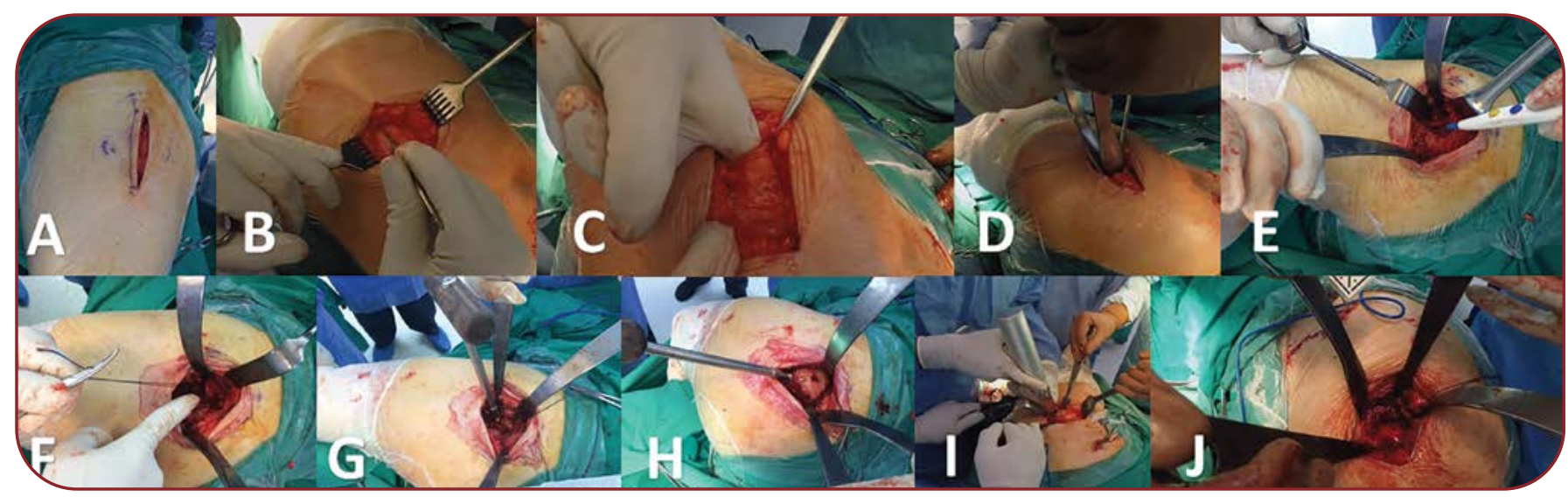

FIGURE 2. A) A longitudinal incision, about $8 \mathrm{~cm}$ is performed right above the greater trochanter region.

B) The aponevrosis of the tensor fascia latae is curved and splitted. C) The interval between tensor fascia latae, gluteus medius and vastus lateralis. D) The interval between tensor fascia latae, gluteus medius and vastus lateralis is exposed with the use of a blunt and then a sharp curved Hohmann retractor. E) Few anterior gluteus medius fibers and gluteus minimus are elevated. F) The few anterior gluteus medius fibers are held with sutures to be controlled during the operation. G, H) Femoral dislocation using careful traction and external rotation by a screwdriver handle (tirebusson). I) Osteotomy of the femoral neck. J) Visualization of the acetabulum during the approach

dislocation is difficult, femoral neck double osteotomy is performed and then femoral head is removed. Hemostatic wax is placed at the osteotomized femoral neck to eliminate any femoral neck bleeding during acetabulum preparation.

At this point, with the limb in external rotation, four sharp Hohmann retractors are placed at the acetabular ring. A curved retractor is placed at about 7 o'clock (or 5 o'clock, depending on the side), declining the femur backwards. Furthermore, retractors are placed at 5 o'clock (or 7 o'clock), 2 (or 10) o'clock and 10 (or 2) o'clock (Figure 2J). The procedures of capsular preparation, osteophyte resection and acetabular preparation are performed in the usual manner, without the use of MIS reamers or special instruments. A threaded cup is positioned usually at about $40-45^{\circ}$ inclination and $7-9^{\circ}$ anteversion (according to preoperative planning).

The lower limb is positioned during femoral exposure in less than $20^{\circ}$ flexion, $20^{\circ}$ adduction and $90^{\circ}$ external rotation in front of the table, over the contra-lateral knee joint and at the place of removed anterior leg support. We recommend the use of curved rasps as well as curved stems, either short or long, depending of age and bone quality, to avoid even greater trochanter or abductors rasping injury. Positioning of implants, following trials, is performed in the usual fashion (Figure 3). The gluteus minimus is reattached at its origin, while the anterior fibers of gluteus me- dius are also reattached. Fascia latae, subcutaneous tissues and skin are closed in the usual manner.

\section{Perioperative care}

All patients received $1 \mathrm{~g}$ of tranexamic acid intravenously (i.v.) dissolved in $100 \mathrm{~mL}$ of N/S 0.9\%, 10 minutes prior incision, while the transfusion

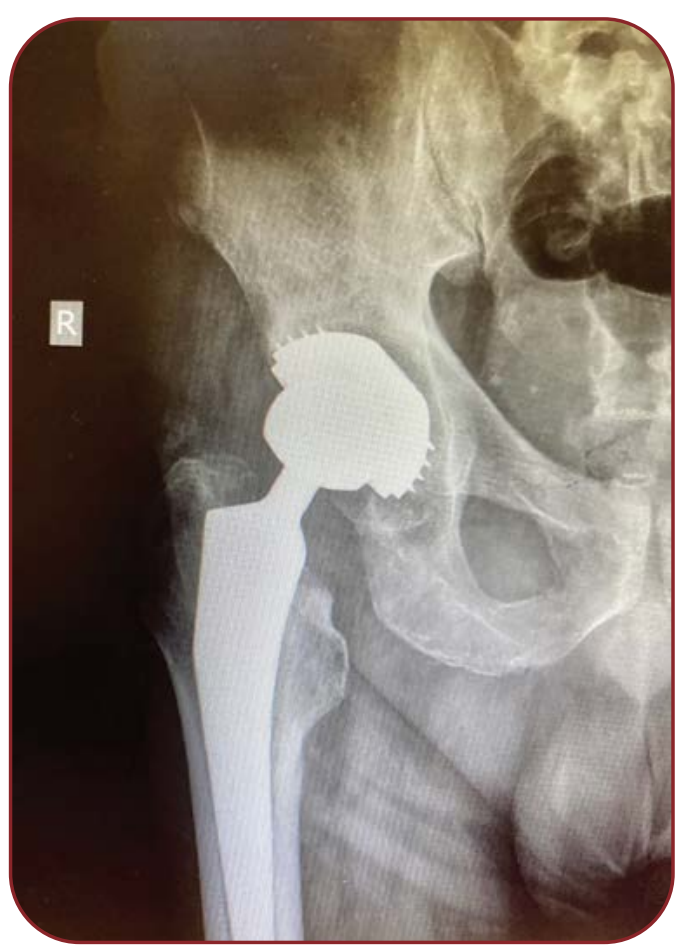

FIGURE 3. Total hip arthroplasty with a curved stem and a threaded cup 
threshold of hemoglobin was $9.5 \mathrm{~g} / \mathrm{dL}$. Postoperatively, a multimodal opioid-sparing analgesia regimen, including gabapentinoids, local infiltration analgesia (ropivacaine $1 \%$ in a maximum dose of $3 \mathrm{mg} / \mathrm{kg}$ ), paracetamol and non-steroidal anti-inflammatory drugs (NSAIDs), was implemented. All patients were also commenced on three doses of $400 \mathrm{mg}$ i.v. teicoplanin (the first dose was received one-hour prior surgery) and sc low molecular weight heparin (starting 12 hours after surgery), continuing for 30 days. Out of bed mobilization was initiated less than 24 hours after surgery, while patients were regularly followed-up in the out patient clinic.

\section{RESULTS}

A total of 154 patients [91 (59.1\%) females and $63(40.9 \%)$ males], with a mean age of 68.9 years [standard deviation (SD) 9.2] at the time of surgery, were included in this study. Ninety three $(60.4 \%)$ patients had primary osteoarthritis, $32(20.8 \%)$ hip dysplasia [high dislocation in $28(18.2 \%)$ cases and low dislocation in four (2.6\%) cases], 15 (9.8\%) osteonecrosis and 14 (9.1\%) post-traumatic arthritis. The mean LOS was 3.2 days (SD 1.4), while the mean follow-up was 53.1 months $(\mathrm{SD}=22.6)$ or 4.6 years (SD 1.9). A total of four (2.6\%) patients required postoperative transfusion with one blood unit each.

Intraoperative complications included greater avulsion trochanter fracture in two $(1.3 \%)$ patients and femur fracture in one $(0.6 \%)$ patient. During the early postoperative period (the first 12 months following surgery), six (3.9\%) patients had superficial hematoma, two (1.3\%) superficial infection treated with empirical antimicrobial treatment, four $(2.6 \%)$ leg lengthening $>1.5 \mathrm{~cm}$ and $12(7.8 \%)$ Trendelenburg sign with a mean of 5.1 degrees (SD 1.1). It is of note that Trendelenburg sign ameliorated during the first three months postoperatively in eight patients, during the first six months in three subjects, while it was permanent in one case. Regarding the late postoperative period, two (1.3\%) cases of dislocation due to a falling event presented, both treated with closed reduction under general anesthesia. Additionally, 12 cases of heterotopic ossification (>Brooker III) were also present.

At final follow-up, a mean Harris hip score of 91.1 (SD 4.4) and a mean satisfaction rate (1-10) of 9.1 (SD 0.7) were found, while no revision surgeries have been performed so far.

\section{DISCUSSION}

T he present study has described in detail the modified by Christodoulou ALMIS approach for THA (3). Furthermore, intraoperative tips and tricks as well as outcomes and complications from 154 cases during a seven-year period have been reported.

The positioning of the patient at the operating table with the posterior support extended 20 degrees and slightly abducted and the anterior leg support removed, as shown in Figure 1, enables better exposure during femoral preparation, avoiding complications such as intraoperative femoral fractures in more difficult cases. Such complications have already been reported during anterolateral minimal invasive hip approaches $(3,6,7)$.

During this modified ALMIS approach, a few anterior fibers of the gluteus medius and gluteus minimus are exposed. The broad, fan-shaped gluteus medius is often functionally subdivided into three sets of fibers: anterior, middle, and posterior $(8,9)$. All fibers contribute to abduction of the hip; however, the anterior fibers also produce a modest internal rotation and the posterior fibers produce extension and external rotation (8-11). The strength and even direction of this muscle's horizontal plane actions can change when the muscle is activated from varying degrees of hip flexion $(10,11)$. At the end of the surgery, the main gluteus muscle mass remains intact. The gluteus minimus tendon is temporary detached backwards under the gluteus medius and then is reinserted using strong Vicryl sutures to its origin at the greater trochanter. Usually, this injury of the gluteus minimus has a minor impact on the clinical outcome, particularly not on the abduction strength, since the main function of the gluteus minimus is rather the centralization of the femoral head in the joint during the gait cycle and participation in internal hip rotation than hip abduction and stabilization of the pelvis $(3,8,12)$. This modified anterolateral MIS hip approach is not only minimally invasive in gluteal muscles but also sparing all significant anatomical elements of the hip, such as the supero-medial or the posterior capsule and the external rotators. 
In our practice, we were utilizing the Hardinge approach for THA. We have experienced problems regarding heterotopic ossification, Trendelenburg gait as well as extensive bleeding and need for transfusion. As years progressed, we tried to perform smaller incisions and less soft tissue damage. With this modified ALMIS approach, the lateral circumflex artery branches in the anterior vastus lateralis, causing most of the bleeding in the Hardinge approach are avoided (13). As recorded in the present cohort, the transfusion rate with this modified ALMIS approach for THA was found to be just $2.6 \%$, while transfusion rates for THA in the literature were ranging between 2 and $70 \%(14,15)$.

The mean Harris hip score at an average follow-up of 4.6 years was found to be 91.1 , while the mean satisfaction rate (1-10) was 9.1. Grading of Harris hip score between 90 and 100 is associated with an excellent outcome, which is also shown by the high subjective satisfaction rate (16). No revisions were needed, while two postoperative dislocation cases were associated with a traumatic event, and not with wrong implant positioning or loosening.

It is of note that all "approach-related" complications were recorded in the first 35 patients of this cohort. These included two greater trochanteric fractures and one femoral fracture, four cases of leg lengthening, 12 cases with Trendelenburg sign and 12 cases with clinically significant (Brooker>III) heterotopic ossification (17). For these first cases, a rectangular femoral stem was used, causing rasping injury to the gluteus medius during femoral preparation ( $\mathrm{Fi}-$

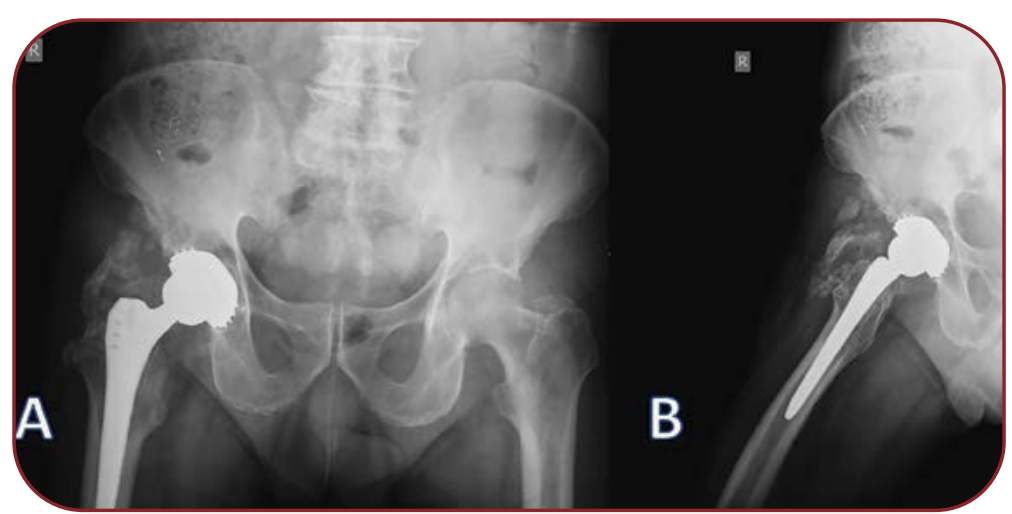

FIGURE 4. A (anteroposterior) and B (lateral) view of case with heterotopic ossification: a rectangular femoral stem was used in this case, causing more rasping injury to the gluteus muscles during femoral preparation gure 4). This was changed to a curved stem, which is what we are currently using (Figure 3). Besides the type of stem that played a role, especially for heterotopic ossification and probably the Trendelenburg sign, the learning curve of such a minimal approach also plays a significant role for minimizing complications and achieving better clinical outcomes $(18,19)$. It is well-documented that great-volume surgeons have better clinical outcomes $(18,19)$.

In particular, regarding the Trendelenburg sign, besides the femoral stem choice, the absence of Trendelenburg gait in the majority of cases is probably related to the careful avoidance of superior gluteus nerve injury branches and protection of the posterior part of gluteus medius, by elevation only a few anterior fibers of the gluteus medius $(1,3,4)$.

The present study has some limitations. It is a retrospective a single center study that does not comprise a control group. Nevertheless, it offers valuable insights regarding this modified ALMIS approach as well as possible pitfalls during its learning curve. Furthermore, the results from a 154-patient cohort during a seven-year period are presented.

\section{CONCLUSIONS}

-his modified ALMIS approach represents a minimal invasive hip approach offering good visibility during acetabular and femoral preparation in THA, as well as excellent clinical results in mid-term follow-up. Like every other surgical approach or technique, it is of utmost importance during the learning curve in order to avoid pitfalls such as rasping injury from rectangular femoral stems, while in extremely difficult cases extension of the incision is possible without jeopardising important anatomical structures such as the lateral cutaneous nerve or the deep femoral artery, which represent the limitations of similar anterior MIS approaches.

Conflicts of interest: none declared

Financial support: none declared.

Authors' contributions: CK, KR, DT for the literature search and analysis and manuscript writing. IK and $K K$ for the final manuscript revision. All authors have read and approved the final manuscript. 


\section{R EFERENCES}

1. Petis S, Howard JL, Lanting BL, Vasarhelyi EM. Surgical approach in primary total hip arthroplasty: anatomy, technique and clinical outcomes. Can J Surg 2015;58:128-139.

2. Migliorini F, Biagini M, Rath B, et al. Total hip arthroplasty: minimally invasive surgery or not? Meta-analysis of clinical trials. Int Orthop 2019;43:1573-1582.

3. Christodoulou N. ALMIS Anterolateral Hip Approach Using a Different Table and Legs Position during Femoral Exposure; New Surgical Technique. MOJ Orthop Rheumatol 2017;7:00282.

4. Bertin $\mathrm{KC}$, Röttinger $\mathrm{H}$. Antero-lateral mini-incision hipreplacement surgery. A modified Watson-Jones approach. Clin Orthop Rel Res 2004;429:248-255.

5. Hansen BJ, Hallows RK, Kelley SS. The Rottinger approach for total hip arthroplasty: technique and review of the literature.

Curr Rev Musculoskelet Med 2011;4:132-138.

6. Kutzner KP, Donner S, Schneider M, et al. One-stage bilateral implantation of a calcar-guided short-stem in total hip arthroplasty: Minimally invasive modified anterolateral approach in supine position. Oper Orthop Traumatol 2017;29:180-192.

7. Hürlimann M, Schiapparelli FF, Rotigliano N, et al. Influence of surgical approach on heterotopic ossification after total hip arthroplasty is minimal invasive better? A case control study.

BMC Musculoskelet Disord 2017;18:27.

8. Tsutsumi M, Nimura A, Akita K. The Gluteus Medius Tendon and Its Insertion Sites: An Anatomical Study with Possible Implications for Gluteus Medius Tears. J Bone Joint Surg Am 2019;101:177-184.

9. Flack NA, Nicholson HD, Woodley SJ. A review of the anatomy of the hip abductor muscles, gluteus medius, gluteus minimus, and tensor fascia lata. Clin Anat 2012;25:697-708.

10. Neumann DA. Kinesiology of the hip: a focus on muscular actions. J Orthop Sports Phys Ther 2010 Feb;40:82-94.

11. Ju SK, Yoo WG. Comparison of anterior gluteus medius fiber activation during general exercises and PNF exercises. J Phys Ther Sci 2017;29:476-477.

12. Jixiang $T$, Hong $C$, Cheng $C$, et al. The strength and function of hip abductors following anterolateral minimally invasive total hip arthroplasty. Chinese Journal of Traumatology. 2014;17(2):73-78.

13. Christodoulou NA, Dialetis KP, Gouzias GK et al. Modified less invasive and bloodless lateral hip approach for total arthroplasty.

Eur J Orthop Surg Traumatol 2012;22:167-174.

14. Lindman IS, Carlsson LV. Extremely Low Transfusion Rates: Contemporary Primary Total Hip and Knee Arthroplasties. J Arthroplasty 2018;33:51-54.

15. Suh YS, Lee JJ, Nho JH, et al. Transfusion trends in hip arthroplasty in Korea: a nationwide study by the Korean National Health Insurance Service. Transfusion 2019;59:2324-2333.

16. Marchetti P, Binazzi R, Vaccari V, et al. Long-term results with cementlessFitek (or Fitmore) cups. J Arthroplasty 2005;20:730-737.

17. Łęgosz P, Sarzyńska S, Pulik $€$, et al. Heterotopic ossification and clinical results after total hip arthroplasty using the anterior minimally invasive and anterolateral approaches. Arch Med Sci 2018;16:613-620.

18. Malik AT, Jain N, Scharschmidt TJ, et al. Does Surgeon Volume Affect Outcomes Following Primary Total Hip Arthroplasty? A Systematic Review. J Arthroplasty 2018;33:3329-3342.

19. Koltsov JCB, Marx RG, Bachner E, et al. Risk-Based Hospital and Surgeon-Volume Categories for Total Hip Arthroplasty. J Bone Joint Surg Am 2018;100:1203-1208. 